\title{
Using Ant Colony Optimisation to Improve the Efficiency of Small Meander Line RFID Antennas
}

\author{
Marcus Randall \\ School of Information Technology \\ Bond University \\ Queensland, Australia \\ mrandall@bond.edu.au
}

\author{
Andrew Lewis \\ Institute for Integrated and Intelligent Systems \\ Griffith University \\ Queensland, Australia \\ a.lewis@griffith.edu.au
}

\author{
Amir Galehdar \\ Centre for Wireless Monitoring and Applications \\ Griffith University \\ Queensland, Australia \\ s2145033@student.griffith.edu.au \\ David Thiel \\ Centre for Wireless Monitoring and Applications \\ Griffith University \\ Queensland, Australia \\ d.thiel@griffith.edu.au
}

\begin{abstract}
Increasing the efficiency of meander line antennas is an important real-world problem within radio frequency identification (RFID). Meta-heuristic search algorithms, such as ant colony optimisation, are very efficient at solving problems that require paths to be constructed. This search technique is adapted to solve the grid based path problem for meander line antennas and incorporates the NEC evaluation suite. The results for grid sizes up to $10 \times 10$ grid indicates that ant colony optimisation is extremely effective at this real-world problem.
\end{abstract}

\section{Introduction}

The idea of radio frequency identification (RFID) was first developed in 1948 [14]. Recently there have been many enhancements to this basic idea and the concept has found applications in many areas. Practical considerations have driven a need for smaller tags with longer reading range. The read range can be explained as "the maximum distance at which RFID reader can detect the backscattered signal from the tag" [12]. This vital factor can be increased by designing antennas with higher gain and this is directly related to the antenna efficiency. Design engineers seek the smallest, most efficient antenna structures for their RFID tags.

As indicated above, one of the key features of RFID is antenna design. Traditional approaches to antenna design are based on simplified electromagnetic calculations which can only approximate real antenna performance. More recently, accurate numerical modelling codes have become available to investigate antenna characteristics before construction. The advent of these codes has allowed antenna design to be improved using mathematical optimisation techniques. For example readers are directed to the Special Issue of IEEE Transactions on Antennas and Propagation vol. 55 (3), March 2007, where many different optimisation techniques for antenna design are reported.

Meander line antennas are a subset of particular interest for RFID as, by their nature, they are compact and tags may be readily manufactured. These antennas normally have a planar structure and consist of printed conductive tracks on thin plastic substrates [5]. While in principle it is possible to fix the size and optimise the track layout in a completely arbitrary manner, the frequency of operation means that the total track length needs to be maximised, the track width needs to be sufficient to ensure adequate conduction, all within the confines of a convenient area. The design of such antennas is quite difficult, not only because of these 
practical constraints, but also because there is no generally applicable analytic design methodology. For this reason, computational methods for design optimisation have proved essential. A few authors have reported approaches using genetic algorithms for the optimisation of antennas for RFID applications $[9,12]$. These have concentrated on simple, serpentine configurations, often regular in structure. There appears to have been little or no systematic investigation of more general configurations and the few undertaken to date $[6,7]$ have not applied computational optimisation, and consequently have been limited in the scale of problem they can tackle.

One approach to design of the general meander line antenna is to confine the antenna to a Cartesian grid. For a particular grid defining the meander line, a number of design choices are possible. This gives rise to a combinatorial optimisation problem similar to the travelling salesman problem (TSP), and as such, can be solved by meta-heuristic search strategies ${ }^{1}$. There has been a large interest in solving TSP using the meta-heuristic ant colony optimisation (ACO) as its mechanics lend themselves naturally to this problem. Ants are agents that iteratively construct solutions and are ideally suited to path planning problems (such as the TSP and vehicle routing problem variants). Despite its apparent suitability, past use of ACO in antenna design has been limited to self-structuring antennas [2]; it has not, to the authors' knowledge, previously been applied to meander line antennas. In this paper, we outline the process and effectiveness for using ACO on the real-world problem of designing fixed-length meander line antennas for RFID devices.

The process of constructing a meander line antenna has similarities to, but is not the same as, two well known problems. As noted, the first of these is the TSP. The aim of the TSP is to find the shortest path to traverse all cities in a given set exactly once and return to the starting city. The major difference between this and the problem defined in this paper is that in the TSP there is a potential connection between each pair of cities, whereas a meander line may only advance to an adjacent (non-diagonal) grid point. The second similar problem is that of producing self-avoiding walks (SAWs) $[8,10,13]$. This variant of the random walk has been profitably applied in chemistry for simulation of linear polymer molecules in a good solvent [13]. As the name suggests, a path is constructed from one point to the next without crossing itself. While this sounds very similar to building meander lines, a SAW generally has an infinite grid size (as polymers exist in continuous space) whereas antennas for RFID applications are strictly confined in their surface area. An exception is the compact polymer problem [10] which closely parallels the problem of constructing general meander lines on a grid. The main difference is

\footnotetext{
${ }^{1}$ This problem is $\mathcal{N} \mathcal{P}$ hard as it is reducible to TSP and vice-versa.
}

that this work considers optimisation of the resultant structure, rather than adequate sampling of the solution space of feasible compact polymers.

The remainder of the paper is organised as follows. Section 2 describes the mechanics of the target ant algorithm for this application, ant colony system (ACS) while Section 3 gives a brief description of meander line antennas as well as an outline of how the meta-heuristic can be used to produce them. Sections 4 and 5 show the computational results and give the conclusions respectively.

\section{The Ant Colony System Algorithm}

ACO is modelled on the foraging behaviour of Argentine ants. The seminal work by Dorigo [3] showed that this behaviour could be used to solve discrete optimisation problems. ACO is in fact a collection of meta-heuristic techniques, one of which is the ant colony system [4]. It is shown to have good performance and is robust enough to be applied across a range of combinatorial optimisation problems. It is used as the basis of the solver described in Section 3. Here we give a brief overview of ACS.

ACS can best be described with the TSP metaphor as it is a well understood optimisation problem and, as previously shown, a close relation to the problem dealt with in this paper. Consider a TSP with $N$ cities. Cities $i$ and $j$ are separated by distance $d(i, j)$. Place $m$ ants randomly on these cities $(m \ll N)$. In discrete time steps, all ants select their next city then simultaneously move to their next city. Ants deposit a substance known as pheromone to communicate with the colony about the utility (goodness) of the edges. Denote the accumulated strength of pheromone on edge $(i, j)$ by $\tau(i, j)$.

At the commencement of each time step, Equations 1 and 2 are used to select the next city $s$ for ant $k$ currently at city $r$. Equation 1 is a greedy selection technique that will choose the city that has the best combination of short distance and large pheromone levels. Using the first branch of Equation 1 exclusively will lead to sub-optimal solutions due to its greediness. Therefore, there is a probability that Equation 2 will be used to select the next closest city instead. This equation generates a probability and then roulette wheel selection is used to generate $s$. These two equations collectively are called the pseudo-proportional rule: $s$ is given by Equation 1 if $q \leq q_{0}$, otherwise it is defined by Equation 2 .

$$
\begin{gathered}
s=\arg \max _{u \in J_{k}(r)}\left\{\tau(r, u)[\eta(r, u)]^{\beta}\right\} \\
s=R\left(\left\{\begin{array}{ll}
\frac{\tau(r, s)[\eta(r, s)]^{\beta}}{\sum_{u \in J_{k}(r)} \tau(r, u)[\eta(r, u)]^{\beta}} & \text { if } s \in J_{k}(r) \\
0 & \text { otherwise }
\end{array}\right)\right.
\end{gathered}
$$


where $\eta(r, u)=d(r, u), R$ is the roulette wheel selection function, $q \in[0,1]$ is a uniform random number and $q_{0}$ is a parameter. To maintain the restriction of unique visitation, ant $k$ is prohibited from selecting a city which it has already visited. The cities which have not yet been visited by ant $k$ are indexed by $J_{k}(r)$. It is typical that the parameter $\beta$ is negative so that shorter edges are favoured. The use of $\tau(r, s)$ ensures preference is given to edges that are well traversed (i.e., have a high pheromone level). The pheromone level on the selected edge is updated according to the local updating rule in Equation 3.

$$
\tau(r, s) \leftarrow(1-\rho) \cdot \tau(r, s)+\rho \cdot \tau_{0}
$$

Where:

$\rho$ is the local pheromone decay parameter, $0<\rho<1$ and

$\tau_{0}$ is the initial amount of pheromone deposited on each of the edges.

Global updating of the pheromone takes place once all ants have constructed a solution. Edges that compose the best solution (so far) are rewarded with an increase in their pheromone level while the pheromone on the other edges is evaporated (decreased). This is expressed in Equation 4.

$$
\tau(r, s) \leftarrow(1-\gamma) \cdot \tau(r, s)+\gamma \cdot \Delta \tau(r, s)
$$

$\Delta \tau(r, s)= \begin{cases}\frac{1}{L} & \text { if }(r, s) \text { is an edge within the best solution } \\ 0 & \text { otherwise. }\end{cases}$

Where:

$\Delta \tau(r, s)$ is used to reinforce the pheromone on the edges of the best solution (see Equation 5),

$L$ is the length of the best (shortest) tour to date and

$\gamma$ is the global pheromone decay parameter, $0<\gamma<$ 1 .

An in-depth pseudocode description of the ACS algorithm can be found in Dorigo and Gambardella [4].

\section{Meander Line Antennas and ACO}

As stated previously, a meander line antenna is one that is laid out on a finite grid of points. To form one line, each point is connected to a point directly horizontal or vertically located. This gives up to four points, with boundary points naturally having fewer than this number. Each point must form part of the meander line. Figure 1 shows a $5 \times 5$ grid as
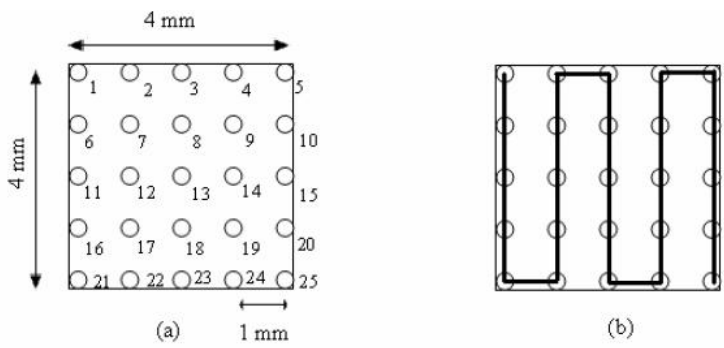

(b)

\section{Figure 1. Diagram (a) defines the grid and its numbering system on a $5 \times 5$ grid. (b) shows a feasible meander line antenna.}

well as one possible solution for the meander line antenna. The feed location is at grid-point 1 .

As in Galehdar et al. [7], some restrictions were set to limit the number of possible structures. Let $n$ be the size of the grid and $s$ be a scaling factor of $4 /(n-1)$. The track grid separation is set at $s \mathrm{~mm}$ and the antenna half-area is set at $s((n-1) \times(n-1)) \mathrm{mm}^{2}$ area with tracks mapped to the $n \times n$ grid points (as shown in Figure 1 for $n=5$ ). The dipole is centre fed along the line of symmetry. That is one half-area was the mirror image of the other half-area. (As such we only need consider the design of one half area. All subsequent references to grid sizes and configurations refer to this "half antenna".) By connecting all grid points, a meander line with the half length of $s\left(n^{2}-1\right) \mathrm{mm}$ is produced. By connecting two half areas together through a $1 \mathrm{~mm}$ bridge (and feed) a dipole meander antenna is formed so the final antenna always occupies $36 \mathrm{~mm}^{2}$ and has total length of $2 s\left(n^{2}-1\right)+1 \mathrm{~mm}$. An additional restriction is that the meander line starts on one of the grid boundary points of the half-area.

There are two important differences between applying ACO to the TSP and this problem. First, the solution will not be in the form of a Hamiltonian circuit. More importantly, not each antenna point neighbours every other point (as would be the case in TSP). Instead, a neighbour is a point that is located one millimeter north, east, south or west on the grid. Points on the border of the grid will number less than four, as there is no wrap-around. Such restrictions make it difficult for ACO (an essentially blind form of search) to construct solutions that include each point of the grid.

The algorithm proceeds as follows. Given a boundary starting point on the grid, each ant chooses a direction in which to move one grid point. Thus up to four neighbours are produced corresponding to the possible horizontal or vertical moves. Neighbours are excluded if they either take the meander line beyond an edge or have already been used 
as part of the line. There are three ways that an ant is guided in the choice of its next direction to travel:

1. Pheromone - The pheromone matrix is an $n^{2} \times 4$ structure. The latter dimension is direction. The pheromone component of the probablistic equations is given as $\tau(c, d)$ where $c$ is the current grid position and $d$ is the direction.

2. Lookahead function - In order to move each ant, the number of unused neighbours of each of the neighbours of the current point is calculated. The greater this number, the higher the probability of choosing that direction

3. Straight line segment function - Encouraging straight line segments helps to ensure that all $n^{2}$ points are visited by the meander line. This is easily calculated by comparing the current candidate direction with the previous direction that the ant has taken.

Modifying equations 1 and 2 to become suitable for this problem simply means that that the heuristic function $\eta$ needs to be replaced by $f(c, d)^{\beta} \times s\left(d, d^{\prime}\right)^{\beta}$ where $f(c, d)$ is the number of free neighbours of position the position given by traversing direction $d$ from current position $c$ and $s\left(d, d^{\prime}\right)$ is 2 if $d=d^{\prime}$ ( $d^{\prime}$ is the previous direction), 1 otherwise.

If the number of neighbours is for an ant at a particular step of the algorithm is 0 , it has become stuck and cannot continue. In this case, the solution is feasible if the length of the meander line is $n-1$ segments, else it is shorter than this required length and is hence discarded. At this point, the ant simply "dies", that is, it is not considered for the remainder of the iteration. Local pheromone updating does occur for the components that these "dead' ants have added to their solution. Fortunately, in ACS this is a form of discouraging other colony members from following the ant. Only the best feasible ant at each iteration may globally (and positively) update the pheromone matrix.

The aim is to maximise the efficiency of the antenna design. However, the calculation of the efficiency of the antenna design is considered as a black box (as it belongs to the NEC [1] antenna suite). Therefore, ants cannot use incremental objective information to guide their paths.

\section{Computational Experiments}

The computing platform used to perform the experiments is a Sun Microsystems v880. The ACS parameter settings are given by the set $\{\beta=2, \gamma=0.1, \rho=0.1, m=$ $\left.10, q_{0}=\{0.9,0.5,0.1\}\right\}$. These values have been found to be robust by Dorigo and Gambardella [4].

The evaluation of meander line antennas is a relatively computationally costly exercise. For our computer platform, while each solution evaluation for a $5 \times 5$ grid requires less than a minute, this increases with grid size and evaluation of a solution on a $10 \times 10$ grid takes 13 minutes. Serial computation of one thousand iterations for the larger grid could take an estimated nine days, an impractical time for delivery of useful results. Given that ants will generate solutions that they have previously come across, it is feasible to implement a solution cache to reduce the total run-time. This cache stores all unique solutions within a run of the solver. If an ant generates one of the solutions in the cache, a look-up on the objective cost value is used (rather than a full evaluation of the antenna design). For the problem size described in this paper, this had the effect of reducing the typical run-time to a more manageable two hours.

For larger grid sizes it is important to use parallel resources to solve these problems in a timely manner. A model to achieve this for ant colony optimisation is outlined in Randall and Lewis [11].

We solved for meander lines on grids ranging from $5 \times 5$ to $10 \times 10$, running the ant algorithm ten times (by varying the random initial seed used for the probabilistic operations of the algorithm). Whilst the $5 \times 5$ problem might be considered a small problem by optimisation standards, it is in fact a useful and practically applicable structure. When the problem is scaled to $10 \times 10$ the number of feasible walks is estimated to rapidly grow to the order of $10^{13}$. The ACS part is coded in the $\mathrm{C}$ language and compiled with $\mathrm{gCC}$. The evaluation of the antennas' efficiencies were simulated by the freely available and widely-used NEC algorithm [1]. Each search was permitted 2000 iterations. The ACO algorithm implemented allows variation of a number of parameters. Of particular interest for the problem considered was the amount of greediness used to select the new grid point (see Equations 1 and 2). This is given by the parameter value of $q_{0}$. A large value favours greedy moves. For each problem size, three different values of $q_{0}$ were used: greedy, non-greedy and an intermediate value. Since each search is entirely independent of all others, parallel computing resources can be utilised to reduce the time to complete the optimisation task.

The results of running the ten ACS searches are summarised in Table 1, which shows the maximum obtained, an estimate of the number of unique solutions evaluated for each search, and the number of iterations required to obtain the maximum. As can be seen in the table, no feasible solutions were obtained for the larger grid sizes, even if the algorithm were allowed to run for a very large number of iterations. To overcome this deficiency a variant of the algorithm was implemented that probabilistically favoured the path of the ants continuing in a straight line. The experiment was repeated using this version of the algorithm and the results are summarised in Table 2. It may be noted that the number of unique solutions considered is an estimate: to the ants, plane rotations of the grid modulo $90^{\circ}$ are 
Table 1. Results of the ACS solver.

\begin{tabular}{lllll}
\hline$q_{0}$ & Grid & Unique & Iterations & Maximum \\
& Size & Solutions & Required & Efficiency\% \\
\hline 0.9 & 5 & 9 & 637 & 83 \\
& 6 & 9 & 1724 & 77 \\
& 7 & 2 & 648 & 56 \\
& 8 & 1 & 137 & 55 \\
& 9 & 0 & $320 \mathrm{k}$ & \\
& 10 & 0 & & \\
\hline 0.5 & 5 & 14 & 10 & 82 \\
& 6 & 9 & 215 & 75 \\
& 7 & 2 & 2477 & 60 \\
& 8 & 0 & & \\
& 9 & 0 & & \\
& 10 & 0 & & \\
\hline 0.1 & 5 & 17 & 323 & 82 \\
& 6 & 11 & 214 & 74 \\
& 7 & 2 & 1979 & 60 \\
& 8 & 0 & & \\
& 9 & 0 & & \\
& 10 & 0 & & \\
\hline & & & &
\end{tabular}

unique, but are considered as identical for the purposes of evaluation when passed to the NEC code.

Overall, the results showed that the ACS search engine is a very effective way of producing good meander line antennas. On all trials the ants produced good solution improvement over time, as shown, for example, in Figure 2. Figure 2 shows the convergence history of one of the searches performed, i.e., the best efficiency obtained to date at each iteration. As may be seen, during some initial period no feasible solutions are generated. But once a feasible path is found it is significantly improved relatively rapidly.

In fact, for the $5 \times 5$ grid one of the searches conducted found a solution extremely close to the global optimum after 637 iterations (approximately 146 seconds of computational time). Due to caching, only 3 unique solutions had to be evaluated for this search. The greatest number requested by any of the searches was 78 . This was achieved in 9 hours, 40 minutes of wall-clock time for the whole experiment.

The total experiment, for a given grid size, consisted of 10 searches with different random seeds for both versions of the algorithm, for each of 3 values of $q_{0}$, a total of 60 independent searches. Due to resource limitations at the time of the experiment only 6 processors were used, searches for different random seeds being processed sequentially. Had additional computational resources been available for this experiment, it would have been a trivial matter to reduce the wall-clock time required by performing independent, parallel searches for the individual random seeds. In addition, as noted above, this time could be reduced further by imple-
Table 2. Results of the ACS solver ("Straightline" variant.)

\begin{tabular}{lllll}
\hline$q_{0}$ & Grid & Unique & Iterations & Maximum \\
& Size & Solutions & Required & Efficiency\% \\
\hline 0.9 & 5 & 7 & 13 & 81 \\
& 6 & 9 & 116 & 77 \\
& 7 & 7 & 182 & 69 \\
& 8 & 6 & 182 & 59 \\
& 9 & 3 & 964 & 49 \\
& 10 & 6 & 921 & 48 \\
\hline 0.5 & 5 & 12 & 138 & 81 \\
& 6 & 10 & 116 & 77 \\
& 7 & 9 & 935 & 70 \\
& 8 & 8 & 479 & 65 \\
& 9 & 5 & 390 & 50 \\
& 10 & 7 & 582 & 49 \\
\hline 0.1 & 5 & 14 & 1904 & 83 \\
& 6 & 9 & 116 & 77 \\
& 7 & 13 & 1822 & 70 \\
& 8 & 10 & 1473 & 67 \\
& 9 & 5 & 1238 & 45 \\
& 10 & 0 & & \\
\hline & & & & \\
\hline
\end{tabular}

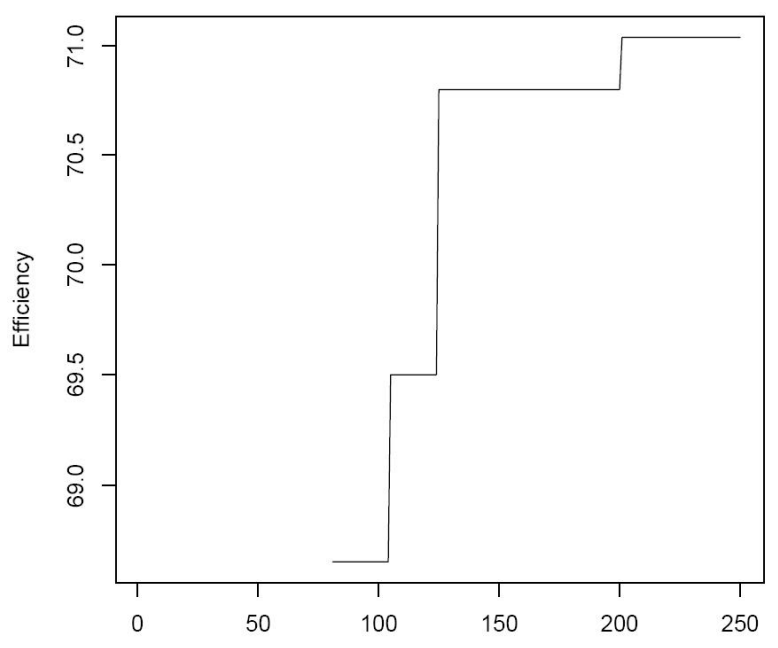

Figure 2. Convergence history of a single run of the solver. 
menting parallel ACO.

Thus our implementation is capable of rapidly finding near-optimal antenna structures as verified by results from exhaustive enumeration in other experiments by Galehdar et al. [6] during which several hundred unique solutions were tested. Scaling to larger grid sizes, enumeration quickly becomes an infeasible method to use to find optimal antenna structures.

Diagrams of the best structures for $5 \times 5$ to $7 \times 7$ grids are shown in Figures 3 to 7. In each case the feed location is in the upper right. Figure 3 shows the structure determined to be the globally optimal configuration by exhaustive enumeration [7]. Figure 4 shows the structure obtained by the ACO search. It may be seen that this latter structure is very similar to the serpentine nature of the globally optimal structure without some refinement. The efficiency of the structure obtained by ACO, at $83 \%$, is within $0.2 \%$ of the global optimum and ranks as one of the best 4 designs.

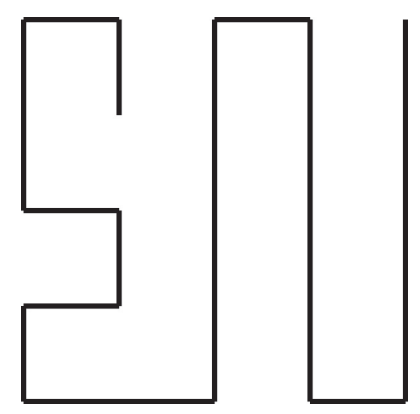

Figure $3.5 \times 5$ globally optimal antenna structure, from Galehdar [7].

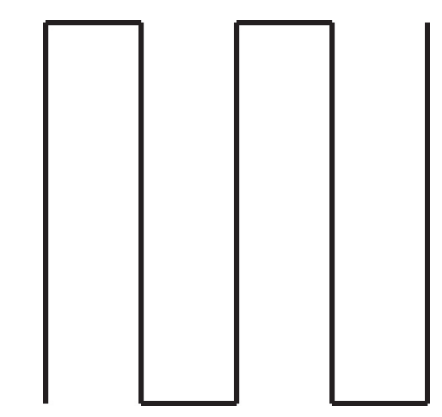

Figure $4.5 \times 5$ best antenna structure from ACO.

From Tables 1 and 2 it can be seen that the best efficiencies for most grid sizes were obtained using the "straightline" variant of the algorithm. For the smaller grid sizes, the "bendy" algorithm was able to obtain solutions of equivalent quality. For the $5 \times 5$ case, both algorithm variants

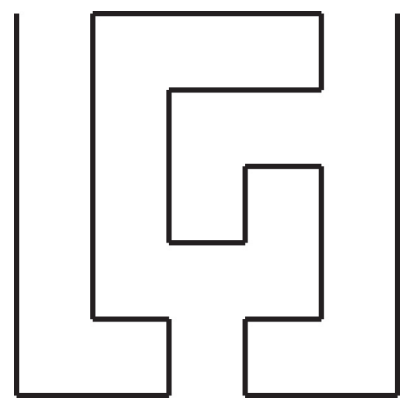

Figure 5. $6 \times 6$ best antenna structure from ACO, "straight-line".

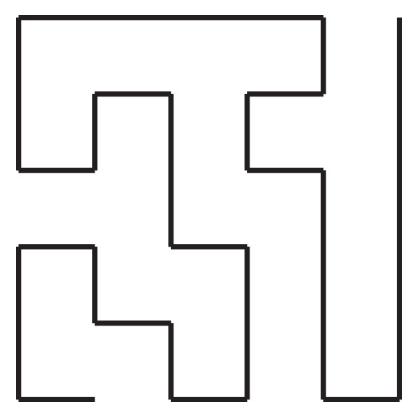

Figure $6.6 \times 6$ best antenna structure from ACO.

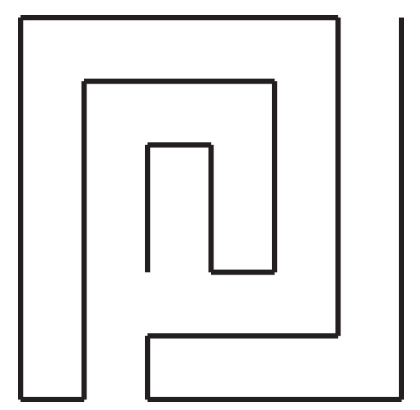

Figure $7.7 \times 7$ best antenna structure from ACO. 
obtained the same structure. Figure 5 shows the structure obtained by the "straight-line" algorithm on the $6 \times 6$ grid, which resembles an "horizontal" serpentine. Figure 6 shows the structure obtained by the "bendy" algorithm, which resembles a "diagonal" serpentine. However, the larger grid sizes only yielded to the "straight-line" algorithm, and most seemed to be more characteristic of spiral structures. Figure 7 shows a form of "bi-folded" spiral previously unexplored. Applying optimisation to the antenna design process had the added benefit of providing insight and novel structures for further investigation.

\section{Conclusions}

Producing efficient meander line antennas is an important design problem for RFID devices. By using a gridaware ACO, we were able to develop an implementation which was able to find a near-optimal antenna design for a $5 \times 5$ antenna grid. This is a notable result as the ants design antennas based purely on a black box evaluator, without having any domain specific knowledge of antenna efficiency.

Given the proof of concept outlined in this paper, we will be working on efficient methods for producing antennas for larger grids. It must be noted that a $10 \times 10$ grid is considered extremely large. There is no known way of solving these in a computationally effective manner. Apart from decreasing the amount of computational time needed for an antenna evaluation, we will be examining parallel processing approaches and techniques of local structure refinement. In addition, this work has focussed on improving antenna efficiency only. Also of importance is the resonant frequency of antenna operation. Future work will be directed to development of multi-objective optimisation methods to simultaneously satisfy both criteria.

\section{References}

[1] G. Burke, A. Poggio, J. Logan, and J. Rockway. NEC - numerical electromagnetics code for antennas and scattering. Antennas and Propagation Society International Symposium, 1979, 17:147-150, 1979.

[2] C. Coleman, E. Rothwell, and J. Ross. Investigation of simulated annealing, ant-colony optimisation and genetic algorithms for self-structuring antennas. IEEE Transactions on Antennas and Propagation, 52(4):1007-1014, 2004.

[3] M. Dorigo. Optimization, Learning and Natural Algorithms. PhD. thesis, Politecnico di Milano, 1992.

[4] M. Dorigo and L. Gambardella. Ant Colony System: A cooperative learning approach to the trav eling salesman problem. IEEE Transactions on Evolutionary Computation, 1:53-66, 1997.

[5] K. Finkenzeller. RFID Handbook: fundamentals and applications in contactless smart cards and identification. Wiley, Chichester, England, 2nd edition, 2003.

[6] A. Galehdar, D. Thiel, and S. O'Keefe. Antenna efficiency calculations for electrically small, RFID antennas. IEEE Antenna and Wireless Propagation Letters, 6:156-159, 2007.

[7] A. Galehdar, D. Thiel, S. O'Keefe, and S. Kingsley. Efficiency variations in electrically small, meander line RFID antennas. In Proceedings of IEEE Antenna Propagation Symposium, 2007.

[8] B. Hayes. How to avoid yourself. American Scientist, 86:314-319, 1998.

[9] G. Marrocco. Gain-optimized self-resonant meander line antennas for rfid applications. IEEE Antennas and Wireless Propagation Letters, 2:302-305, 2003.

[10] R. Oberdorf, A. Ferguson, J. Jacobsen, and J. Kondev. Secondary structures in long compact polymers. Physical Review E, 74(5 Pt 1):051801. Epub, 2006.

[11] M. Randall and A. Lewis. A parallel implementation of ant colony optimization. Journal of Parallel and Distributed Computing, 62:1421-1432, 2002.

[12] K. Seshagiri Rao, P. Nikitin, and S. Lam. Antenna design for UHF RFID tags: A review and a practical application. IEEE Transactions on Antennas Propagation, 53:3870-3876, 2005.

[13] A. Sokal. Monte carlo methods for the self-avoiding walk. Nuclear Physics B Proceedings Supplement, 47:172-179, 1996.

[14] H. Stockman. Communication by means of reflected power. In Proceedings of the Institute of Radio Engineers, pages 1196-1204, 1948. 